\title{
EFOMP and EANM: joint recommendations for a curriculum for the education and training of physicists in nuclear medicine
}

\author{
M. N. Lonsdale • M. Bardiès • R. Boellaard • \\ S. Christofides • G. Flux • M. Lassmann • B. Sattler • \\ A. Del Guerra
}

Published online: 9 February 2013

(C) Springer-Verlag Berlin Heidelberg 2013

\section{Physicists in nuclear medicine: the current situation in Europe}

Physicists working in nuclear medicine clinics and departments across Europe constitute a rather heterogeneous mix of individuals. Whilst many have a degree in medical physics or biophysics, others have initial training in areas such as particle physics and a substantial number have been trained as engineers. Valuable skills learned in these fields can be translated to clinical research and development, particularly with regard to imaging hardware and image processing. Others have a Ph.D. with extensive research experience. Staffing levels, responsibilities, working areas and position

\footnotetext{
M. N. Lonsdale ( $\triangle)$

Department of Clinical Physiology and Nuclear Medicine, Bispebjerg Hospital, Bispebjerg Bakke 23/KFNM,

2400 Copenhagen, Denmark

e-mail: Markus.Lonsdale@regionh.dk

M. Bardiès

Centre de Recherche en Cancérologie de Toulouse,

UMR 1037 INSERM/UPS,

Toulouse, France

R. Boellaard

Department of Nuclear Medicine and PET Research, VU

University Medical Centre, Amsterdam, The Netherlands

S. Christofides

Medical Physics Department, Nicosia General Hospital,

Nicosia, Cyprus

G. Flux

Joint Department of Physics, Royal Marsden Hospital \& Institute of Cancer Research, London, UK
}

in the organisation and its hierarchy vary according to local organisational arrangements, national regulations (or the lack thereof) and other factors.

Today, most if not all countries have a well-defined route into medical physics. This is usually a Bachelor in Physics, followed by a Master in Medical Physics and finally an internship focussing on practical training in parallel with further theoretical education. There is, however, considerable variation in this last part. Some countries have established schools of medical physics linked to universities, in other countries the national societies for medical physics have collected a curriculum that must be worked through in parallel with the internship at a recognised department

\footnotetext{
M. Lassmann

Department of Nuclear Medicine, University of Würzburg, Würzburg, Germany

B. Sattler

Department of Nuclear Medicine, University Hospital Leipzig, Leipzig, Germany
}

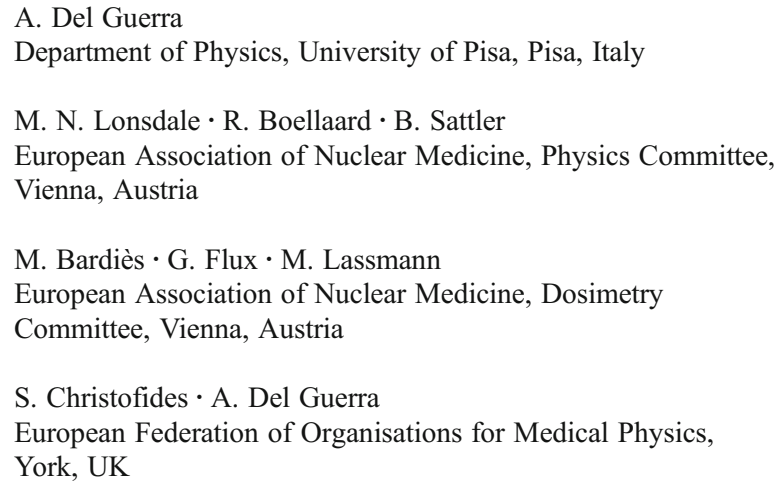


under guidance of a competent, approved mentor. In other countries a formal postgraduate education as medical physicist (MP) is just about to be established.

Thus, comparison of the various national educational systems leading to a university degree in physics (or engineering) - and from there to a further qualification for working in a hospital - is not an easy task. Therefore, physicists moving to another European country often face difficulties when trying to persuade a possible future employer about their qualifications. Vice versa, hospitals in need of physicists may not be able to employ competent applicants who were trained in another country, as their qualifications are not necessarily recognised by the authorities in the receiving country. Examples of these obstacles were revealed in a recent survey [1]. This situation is not only unfortunate for the individual. At a European level, this lack of mutual recognition presents a serious problem as it prevents mobility within Europe, which is to the benefit of all countries.

\section{Formal definitions}

Some of these barriers can be explained by the fact that there is no definition of the MP that is consistently recognised on a European level. This is somewhat surprising as there are wellknown definitions issued by the scientific societies, for example the International Organization for Medical Physics (IOMP):

Medical physicists are professionals with education and specialist training in the concepts and techniques of applying physics in medicine. Medical physicists work in clinical, academic or research institutions. Medical physicists working in the clinical environment are health professionals, with education and specialist training in the concepts and techniques of applying physics in medicine, competent to practice independently in one or more of the sub-fields (specialties) of medical physics.

This rather clear definition has so far not found its way to documents issued by the European Union (EU) and the national regulatory authorities. In two directives stemming from the EURATOM treaty, the Commission has outlined roles for physicists working with radiation protection. The "MED directive" [2] addresses radiation protection of patients, carers and the public from medical exposures and defines somewhat vaguely a "medical physics expert" (MPE). Due to the lack of clarity, the implementation of this title varies considerably across Europe. The other directive, "the EU Basic Safety Standards (BSS) Directive" [3], concerned with radiation protection in general, had previously introduced another title for persons with a similar role (but outside the medical field): the "qualified expert", in later documents also called "radiation protection expert". Unfortunately, the roles defined in the two directives are not consistent and this still causes confusion and misunderstandings.

Fortunately, a new directive ("Proposal for a Council Directive laying down basic safety standards for protection against the dangers arising from exposure to ionising radiation [COM(2012)242]", http://ec.europa.eu/energy/ nuclear/radiation_protection/doc/2012_com_242.pdf, dated 30.5 .2012 ) is currently under revision. It is meant to replace the former two directives and will also provide a clearer definition for an MPE: "medical physics expert means an individual having the knowledge, training and experience to act or give advice on matters relating to radiation physics applied to medical exposure, whose competence to act is recognised by the competent authorities" (article 4, definition \#40 in the draft document).

Comparing the definitions provided by IOMP and the Commission it becomes clear that being an MPE (as defined in the directive) is an important role of an MP (as defined by IOMP). This is especially true in nuclear medicine, where most activities are related to the use of ionising radiation. However, the scope of work of an MP is considerably wider. Thus, an MPE should not per se be regarded as a senior and experienced $\mathrm{MP}$ - despite the expert in the acronym. On the other hand, being an MPE requires a high level of expertise with respect to application of ionising radiation-probably more expertise than the average MP would have.

On this rather confusing background it seems more than logical that some sort of harmonisation would be helpful. This was a major motivation for the European Federation of Organisations for Medical Physics (EFOMP) and the European Association of Nuclear Medicine (EANM) to set out to collect and compose a modern curriculum specifically for the education and training of medical physicists in nuclear medicine. EFOMP (http://efomp.org) is the umbrella organisation for the national medical physics organisations and societies. One of the main objectives of EFOMP is the harmonisation and promotion of the best practice of medical physics in Europe. For this purpose, EFOMP issues policy statements on various topics, for example on the "Continuing Professional Development for the Medical Physicist", describing the planned acquisition of qualifications required for professional practice throughout a working life. Likewise, the EANM-representing nuclear medicine in Europe-aims at advancing science and education in nuclear medicine for the benefit of public health. Physicists from both the Dosimetry Committee and the Physics Committee of the EANM joined EFOMP in the effort of composing the curriculum. Similar scientific collaborations were established by EFOMP with the European Society of Radiology (ESR) and the European Society for Radiotherapy and Oncology (ESTRO) for a curriculum in radiology [4] and radiation oncology [5], respectively. 


\section{Terminology and concepts for definition of educational and training activities}

Modern curricula should follow the conventions laid out by the European Commission. In particular, they should be in line with the Bologna declaration, the tuning process for the educational structures in Europe and the recommendation on the establishment of the European qualifications framework for lifelong learning. Learning outcomes of educational and training programmes are expressed in terms of:

- Knowledge - covering facts, principles, theories and practices

- Skills-meaning the ability to use knowledge and know-how to complete tasks and solve problems (cognitive skills as well as practical skills including the use of methods, materials, tools and instruments)

- Competence-meaning responsibility and autonomy

On first encounter, these terms may appear confusing. But after dealing with them for a while, the underlying concept reveals its advantages. It is also important to note that there is focus on the learning outcome, not the teaching content or the teaching form. Learning outcomes can be divided into two categories: generic and subject-specific. Generic learning outcomes are transferable across all academic professions (e.g. "extract knowledge from various sources") and will not be considered further. Subjectspecific learning outcomes are those that are specific to the profession, here the MP, and are those listed in the curriculum.

An example may help to illustrate the concept: The subject-specific learning outcomes for physicists are subdivided into 11 key activities (listed further below). For each key activity, a catalogue of required knowledge, skills and competences (KSC) is listed (see also Table 1).

\section{Curriculum for medical physicists in nuclear medicine}

The curriculum for medical physicists in nuclear medicine is made up of four main sections, each covering a specific aspect of the work of an MP:

- Physical scientist - fundamental physics knowledge and measurement skills expected of all physicists.

- Healthcare professional-fundamental knowledge for working in the healthcare sector.

- Clinical medical device/physical agents expert-common learning outcomes relevant for medical physicists working in any sub-speciality covering medical devices and safety from associated physical agents. This section is further subdivided into 11 key activities.
Table 1 Example of a catalogue of KSCs: the key activity "physical agents dosimetry service" deals with dosimetry of various physical agents. A catalogue of required KSCs is listed. For brevity, only two items are listed in each category

Catalogue of KSCs

Knowledge

- List and explain the statutory and institutional role of Medical Physics Services with respect to dosimetry of physical agents (including ionising radiation from sealed/unsealed sources)

Define and explain the physical quantities used to assess beneficial or adverse biological effects for the various types of physical agents (including ionising radiation) and methods for their measurement

$\cdot \ldots$

Skills

- Select and operate instrumentation for the measurement of the physical quantities used to assess beneficial or adverse biological effects for the various types of physical agents for the patients, workers and public in own area/s of medical physics practice

Operate radiation measurement sensors/detectors and interpret the results

$\cdot \ldots$

\section{Competences}

- Oversee the installation of a laboratory for the measurement of quantities used to assess beneficial or adverse biological effects for the various types of physical agents for the patients, workers and public in own area/s of medical physics practice

- Take responsibility for the selection and operation of instruments for the measurement of the physical quantities used to assess beneficial or adverse biological effects for physical agents in own area/s of medical physics practice

...

- Nuclear medicine physics - detailed learning outcomes specifically for medical physicists in nuclear medicine subdivided into the same 11 key activities.

The subdivision into 11 key activities (each listing a catalogue of KSCs) illustrates important areas of responsibility for MPs:

- Comprehensive physics problem solving service

- Physical agents dosimetry service (exemplified in Table 1)

- Patient safety/risk management

- Occupational \& public safety/risk management

- Clinical medical device management

- Clinical involvement

- Development of service quality

- Expert consultancy

- Education of healthcare professionals and medical physics trainees

- Economic issues/health technology assessment

- Innovation

The reason for duplicating these key activities in the sections "clinical medical device/physical agents expert" and 
"nuclear medicine" is to facilitate career opportunities for people moving from one field of medical physics to another. An MP would only have to learn the learning outcomes specific for the new field.

The curriculum does not only describe the required KSCs needed to accommodate today's needs in modern nuclear medicine. Also addressed are areas like organisational competence, professionalism, communication, collaboration and social actions. In addition, the role of a medical physicists in nuclear medicine is explicitly stated, namely to

.. provide critical scientific input on the physical processes and technology that underpin the whole nuclear medicine pathway. (..) Nuclear medicine physicists design and develop the framework of radionuclide handling and delivery, radiopharmaceutical dosimetry, optimisation, quality assurance of individual patient's treatments and of the medical imaging equipment, including radiation safety of the patient and staff. Specifically, the nuclear medicine physicist provides expert advice on the development, implementation and improvement of medical imaging with radionuclides and the treatment of benign or malignant diseases with radiopharmaceuticals. The nuclear medicine physicist's role is key to the enabling of the practice of safe, stateof-the-art nuclear medicine diagnosis and treatment (introductory section of [6]).

Clearly, listing the complete curriculum would take up a considerable amount of space in this journal. The purpose of this editorial is to draw the community's attention to the document. As the concept of KSCs still is not widely known, we also want to share the underlying thoughts and philosophy. The curriculum is published in its complete version in Physica Medica: European Journal of Medical
Physics [6]. In addition, the curriculum will be available for download from the EANM website (http://eanm.org, Guidelines section).

It is our hope that the curriculum will find a large readership and widespread application. Our intention was to provide a document that will help to harmonise national training and recognition schemes in the EU and beyond, eventually for the benefit of patients and society as a whole. National authorities and scientific societies should use the curriculum as a basis for new curricula or to update existing curricula. We have tried not only to include classical methodology but also to incorporate current trends in such a way that the learning outcomes will remain relevant for many years.

\section{References}

1. Guibelalde E. Guidelines on Medical Physics Expert Surveys. http:// portal.ucm.es/web/medical-physics-expert-project/inicio. 2012.

2. Council of the European Union. Council Directive 97/43/Euratom of 30 June 1997 on health protection of individuals against the dangers of ionising radiation in relation to medical exposure. 1997.

3. Council of the European Union. Council Directive 96/29/Euratom of 13 May 1996 laying down basic safety standards for the protection of the health of workers and the general public against the dangers arising from ionising radiation. 1996.

4. EFOMP, ESR: Core curriculum for medical physicists in radiology. 2011. Available via the EFOMP website: http://www.efomp.org/ professional-issues/policy-statements.html

5. Eriksen JG, Beavis AW, Coffey MA, Leer JWH, Magrini SM, Benstead K, et al. The updated ESTRO core curricula 2011 for clinicians, medical physicists and RTTs in radiotherapy/radiation oncology. Radiother Oncol 2012;103:103-8.

6. Del Guerra A, Bardies M, Belcari N, Caruana CJ, Christofides S, Erba $\mathrm{P}$, et al. Curriculum for education and training of Medical Physicists in Nuclear Medicine: recommendations from the EANM Physics Committee, the EANM Dosimetry Committee and EFOMP. Phys Med 2012; http://dx.doi.org/10.1016/j.ejmp.2012.06.004. 